\title{
Níveis inatos de C3 e C4 em pacientes curados para a leishmaniose visceral e associação com os aspectos clínico-laboratoriais
}

\author{
Innate levels of $\mathrm{C} 3$ and $\mathrm{C4}$ in patients cured for visceral leishmaniasis and association \\ with clinical and laboratory aspects.
}

Ana Rubia Alcantara Pelloso ${ }^{1}$, Amanda Aparecida Silva de Aguiar ${ }^{2}$, Paloma Barbosa Fernandes ${ }^{1}$, Luis

Fernando Baldino Lopez ${ }^{3}$, Luiz Euribel Prestes Carneiro ${ }^{4}$, Eliana Peresi Lordelo ${ }^{5 *}$

\begin{abstract}
${ }^{1}$ Bacharel em Biomedicina pela Universidade do Oeste Paulista - Unoeste; ${ }^{2}$ Mestre em Ciências da Saúde pela Universidade do Oeste Paulista - Unoeste; ${ }^{3}$ Mestrando do Curso de Mestrado em Ciências da Saúde da Universidade do Oeste Paulista - Unoeste; ${ }^{4}$ Doutor em Imunologia pela Universidade de São Paulo - USP, Docente do Curso de Mestrado em Ciências da Saúde e do Programa de Pós-graduação em Meio Ambiente e Desenvolvimento Regional da Universidade do Oeste Paulista - Unoeste; ${ }^{5}$ Doutora em Doenças Tropicais pela Faculdade de Medicina de BotucatuUNESP, Docente do Curso de Mestrado em Ciências da Saúde da Universidade do Oeste Paulista - Unoeste
\end{abstract}

\begin{abstract}
Resumo
Introdução: assim que inoculada pelo flebotomíneo, a Leishmania entra em contato com o sistema complemento, sendo que poucos estudos têm avaliado os níveis inatos dos componentes iniciais C3 e C4. Objetivo: avaliar os níveis inatos dos componentes C3 e C4 do sistema complemento em pacientes curados de leishmaniose visceral (LV) e sua associação com aspectos clínico-laboratoriais no momento de diagnóstico da doença. Metodologia: foram estudados 29 pacientes com LV curada. Os níveis de C3 e C4 séricos foram dosados pela técnica de imunodifusão radial simples, após um tempo médio de 59,48 meses pós-tratamento, formados os grupos: C3: baixo (< $84 \mathrm{mg} / \mathrm{dl} ; \mathrm{n}=10)$, normal ( 84 a $193 \mathrm{mg} / \mathrm{dl} ; \mathrm{n}=14$ ) e elevado ( $>193 \mathrm{mg} / \mathrm{dl} ; \mathrm{n}=5) ; \mathrm{C} 4$ : muito baixo $(<20 \mathrm{mg} / \mathrm{dl} ; \mathrm{n}=10)$, baixo (20 a $40 \mathrm{mg} / \mathrm{dl} ; \mathrm{n}=15$ ) e normal (> $40 \mathrm{mg} / \mathrm{dl} ; \mathrm{n}=4$ ). Os dados clínicos e laboratoriais empregados para as análises foram coletados por levantamento dos prontuários, considerando o período de diagnóstico da doença de cada paciente. Resultados: foi observada uma correlação positiva fraca entre os níveis de C3 e C4 ( $r h o=0,46 ; p=0,01)$. Verificou-se que a maioria dos pacientes sintomáticos no momento do diagnóstico apresentavam níveis inatos normais de C3 e baixos de C4. Pacientes com C3 baixo apresentaram maiores níveis do hematócrito em relação ao grupo C3 normal $(p=0,0406)$. Conclusão: conclui-se que o componente C3 do sistema complemento está associado às alterações do hematócrito, sugerindo o acompanhamento dos seus níveis em pacientes com LV. Palavras-chave: Leishmania. Proteínas do Sistema Complemento. Sinais e sintomas. Testes Sorológicos
\end{abstract}

\begin{abstract}
Introduction: once inoculated by the sand fly, Leishmania comes into contact with the complement system, and few studies have evaluated the innate levels of the initial components C3 and C4. Objective: to evaluate the innate levels of the C3 and C4 components of the complement system in patients cured of visceral leishmaniasis (VL) and its association with clinical and laboratory aspects at the time of diagnosis of the disease. Methodology: twenty-nine patients with cured VL were studied. Serum C3 and C4 levels were measured by simple radial immunodiffusion technique, after an average time of 59.48 months post-treatment, forming the groups: C3: low (<84 $\mathrm{mg} / \mathrm{dl} ; n=10)$, normal (84 to $193 \mathrm{mg} / \mathrm{dl} ; n=14$ ) and high (> $193 \mathrm{mg} / \mathrm{dl} ; n=5) ; C 4:$ very low (<20 mg/dl; $n=10)$, low (20 to $40 \mathrm{mg} / \mathrm{dl} ; n=15)$ and normal (> $40 \mathrm{mg} / \mathrm{dl} ; \mathrm{n}=4$ ). The clinical and laboratory data used for the analyzes were collected by surveying the medical records, considering the period of diagnosis of the disease of each patient. Results: a weak positive correlation was observed between C3 and $C 4$ levels (rho=0.46; $p=0.01$ ). Most symptomatic patients at the time of diagnosis were found to have normal C3 and low C4 levels. Low $C 3$ patients had higher levels of hematocrit compared to the normal C3 group ( $p=0.0406)$. Conclusion: in conclusion, the C3 component of the complement system is associated with changes in the hematocrit, suggesting the monitoring of its levels in patients with VL. Keywords: Leishmania. Complement System Proteins. Signs and Symptoms. Serologic Tests
\end{abstract}

\section{INTRODUÇÃO}

A leishmaniose é uma doença infecciosa causada por parasitas do gênero Leishmania, cuja letalidade sem tratamento pode alcançar até $10 \%{ }^{1}$. Existem três manifestações clínicas da doença: visceral (apresenta uma maior

Correspondente/ Corresponding: *Eliana Peresi Lordelo - Universidade do Oeste Paulista - Unoeste - End: Rod. Raposo Tavares km 572, CEP 19067-175 Presidente Prudente - SP - Brazil - Tel: (18) 3229-1289 Email: elianaperesi@yahoo.com.br gravidade); cutânea (mais encontrada); mucocutânea. Nas Américas, são transmitidas entre os animais e o homem por intermédio da picada das fêmeas de espécies distintas de flebotomíneos, pertencentes ao gênero Lutzomyia ${ }^{2}$.

A resposta imune inicia pela ativação da imunidade inata, que possui como componentes celulares os fagócitos (neutrófilos, monócitos e macrófagos), células que liberam mediadores inflamatórios (basófilos, mastócitos e eosinófilos) e células natural killers. Os componentes 
solúveis incluem o sistema complemento, as proteínas de fase aguda e as citocinas ${ }^{3}$. O sistema complemento é um conjunto de mais de 30 proteínas solúveis no plasma sanguíneo que estão envolvidas em uma rápida eliminação de patógenos ${ }^{4}$. Existem três vias de ativação do sistema complemento: clássica, das lectinas e alternativa. A via clássica interage com anticorpos ligados na superfície do patógeno, enquanto a ligação com lectina ligante de manose (MBL) e ficolinas ativam a via das lectinas ${ }^{5}$. Ao contrário das vias anteriormente citadas, a via alternativa pode ser ativada diretamente pelo patógeno ${ }^{6}$.

A Leishmania é um parasita intracelular obrigatório, portanto necessita de um nicho intracelular para sobreviver e se multiplicar, entretanto, assim que as promastigotas são inoculadas pelo flebotomíneo na pele, o parasita entra em contato com o sistema complemento e, apesar de algumas controvérsias sobre seu papel na eliminação da Leishmania, já foi demonstrado o envolvimento das três vias neste processo ${ }^{5}$.

A participação da via alternativa na leishmaniose visceral (LV) e cutânea foi demonstrada através da queda da fração de $\mathrm{C} 3$ em associação com a $\mathrm{LV}^{7}$ e de níveis mais elevados de $\mathrm{C} 3$ em relação aos de $\mathrm{C} 4$ em ambos os grupos estudados ${ }^{8}$. Em estudo experimental foi comprovada a ativação do sistema complemento pela Leishmania, promovendo a opsonização do parasita, principalmente pelo componente $\mathrm{C} 3{ }^{9}$.

Por outro lado, a deficiência de C3 promove o desenvolvimento de doenças autoimunes, infecções ou ambas. As infecções tendem a ser recorrentes e severas. Infecções recorrentes, como pela Neisseria meningitidis, foram associadas à deficiência do sistema complemento e sugerido que estes pacientes sejam avaliados para diferentes componentes do sistema complemento ${ }^{10}$, entretanto, não foram encontrados estudos que avaliaram esta relação em pacientes com leishmaniose.

Diversas interações parasita-hospedeiro têm sido estudadas na tentativa de compreender melhor o desenvolvimento da leishmaniose, entretanto poucos estudos têm avaliado os níveis constitutivos dos componentes iniciais do sistema complemento, e se estes níveis colaboram ou não para a permanência ou eliminação do parasita. Desta forma, o objetivo deste trabalho foi avaliar os níveis inatos dos componentes $\mathrm{C} 3$ e C4 do sistema complemento em pacientes curados de LV e sua associação com aspectos clínico-laboratoriais no momento de diagnóstico da doença.

\section{METODOLOGIA}

Foram estudados 29 pacientes, dentre os quais 18 homens, com média de idade de 51 anos (mínima 19 anos; máxima 76 anos) e 11 mulheres, com média de idade de 43 anos (mínima 20 anos; máxima 82 anos), atendidos no Ambulatório de Infectologia do Hospital Regional de Presidente Prudente/SP, com diagnóstico de LV comprovado por quadro clínico-epidemiológico e/ ou diagnóstico imunológico, por teste imunoenzimático
(ELISA) ou Imunofluorescência indireta (IFI). No momento da coleta de sangue para dosagem dos níveis inatos dos componentes $\mathrm{C} 3$ e C4 do sistema complemento, todos os pacientes eram considerados curados para a LV e, haviam completado o tratamento com média de tempo de 59,48 meses (mínimo 12 meses; máximo 108 meses). Os dados clínicos e laboratoriais empregados para as análises foram coletados por levantamento dos prontuários, no período de diagnóstico da doença de cada paciente.

Os componentes $\mathrm{C} 3$ e $\mathrm{C} 4$ séricos foram dosados pela técnica de imunodifusão radial simples, conforme as normas do fabricante (DIFFU-PLATE, Biocientífica). Brevemente, foram colocados $5 \mu \mathrm{l}$ de soro de cada amostra em um poço da placa contendo anticorpos anti-C3 ou anti-C4 e incubados durante 48 horas. Após o período de incubação, o diâmetro do halo formado ao redor do poço foi medido em milímetros e a concentração determinada através de valores preestabelecidos na bula do kit. O valor médio de referência para a dosagem de C3 foi de 138 $\mathrm{mg} / \mathrm{dl}$, com intervalo de confiança de $95 \%$ entre 84 e 193 $\mathrm{mg} / \mathrm{dl}$, desta forma, os participantes foram divididos em três grupos: baixo (<84 mg/dl; $n=10)$, normal ( 84 a 193 $\mathrm{mg} / \mathrm{dl} ; \mathrm{n}=14$ ) e elevado ( $193 \mathrm{mg} / \mathrm{dl} ; \mathrm{n}=5$ ). Para o $\mathrm{C} 4$, o valor médio de referência foi de $28 \mathrm{mg} / \mathrm{dl}$, com intervalo de confiança de $95 \%$ entre 20 e $40 \mathrm{mg} / \mathrm{dl}$, desta forma, os participantes foram divididos em três grupos: muito baixo ( $<20 \mathrm{mg} / \mathrm{dl}$; $\mathrm{n=10}$ ), baixo (20 a $40 \mathrm{mg} / \mathrm{dl} ; \mathrm{n}=15)$ e normal (> $40 \mathrm{mg} / \mathrm{dl} ; \mathrm{n}=4$ ).

Para a avaliação da gravidade da LV foram utilizados três critérios: hepatomegalia, esplenomegalia e disfunção hepática (TGO > $100 \mathrm{U} / \mathrm{l}$ e/ou TGP > $100 \mathrm{U} / \mathrm{l}$ ). Os pacientes foram classificados com LV grave na presença de pelo menos dois dos três critérios utilizados. Foi realizada uma análise descritiva da distribuição da frequência relativa (\%) dos sintomas. As porcentagens dos sintomas em cada grupo foram calculadas considerando o número de indivíduos que apresentaram o sintoma em relação ao número total de participantes $(n=29)$ e, cada porcentagem foi subdividida segundo os níveis de C3 e C4. A associação dos componentes $\mathrm{C} 3$ e C4 com a gravidade da LV foi analisada pelo teste do $\chi^{2}$. A associação dos componentes $\mathrm{C} 3$ e $\mathrm{C} 4$ com as variáveis laboratoriais foi analisada pelo teste de Kruskal-Wallis, seguido pelo teste de Dunn. A correlação entre os níveis de C3 e C4 foi realizada pelo teste de Spearman. Em todas as análises foi considerado significativo valores de $p<0,05$.

Todos os pacientes foram informados sobre o estudo e assinaram termo de consentimento livre e esclarecido. A pesquisa foi aprovada pelo Comitê de Ética em Pesquisa da Universidade do Oeste Paulista (CAAE: 95040318.0.0000.5515) e está de acordo com a Declaração de Helsinki de 1964.

\section{RESULTADOS}

Os níveis inatos de $\mathrm{C} 3$ e $\mathrm{C} 4$ de pacientes curados para $\mathrm{LV}$ apresentaram uma correlação positiva fraca ( $\mathrm{rho}=0,46$; $p=0,01$ ) (Figura 1). 
Figura 1 - Correlação dos níveis inatos de C3 e C4 em pacientes com leishmaniose visceral curada. A correlação foi realizada pelo teste de Spearman. Foi considerado significante $p<0,05$.

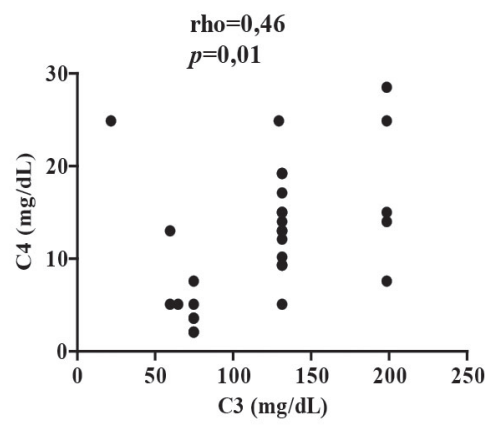

Dentre os sintomas apresentados no momento do diagnóstico, os mais frequentes foram a febre $(n=26)$, hepatomegalia $(n=25)$, esplenomegalia $(n=22)$ e perda de peso $(n=12)$. Quando avaliamos a distribuição dos sintomas segundo os níveis inatos de C3, verificamos que a maioria dos pacientes com LV apresentava níveis normais, enquanto os níveis de C4 se apresentavam baixos (Figura 2). Quando classificamos os pacientes segundo a gravidade da LV, foi verificado um maior número de indivíduos graves $(n=24)$, característica que não foi influenciada pelos diferentes níveis de C3 e de C4 (Tabela 1).

Fonte: Dados da pesquisa.

Figura 2 - Distribuição dos sintomas presentes nos pacientes com leishmaniose visceral curada segundo a classificação dos níveis inatos de C3 e C4. Cada barra refere-se à frequência relativa (\%) de pacientes que apresentaram o sintoma em relação ao número total $(n=29)$.

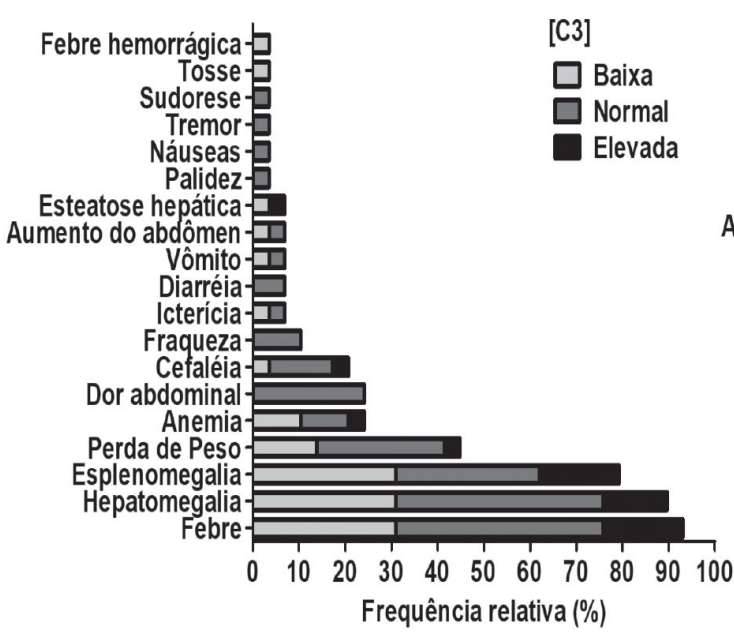

Fonte: Dados da pesquisa.

Tabela 1- Associação da gravidade da leishmaniose visceral com os niveis inatos de C3 e C4 de pacientes com leishmaniose visceral curada $(n=29)$, segundo o teste do $\chi^{2}$.

\begin{tabular}{llccc}
\hline Classificação níveis inatos & Não grave (n) & Grave (n) & $p$ \\
\hline C3 & Baixo & 2 & 8 & \\
& Normal & 2 & 11 & 0,9507 \\
& Elevado & 1 & 4 & \\
C4 & Muito Baixo & 1 & 9 & \\
& Baixo & 1 & 11 & 0,0676 \\
& Normal & 3 & 3 & \\
\hline
\end{tabular}

Fonte: Dados da pesquisa.

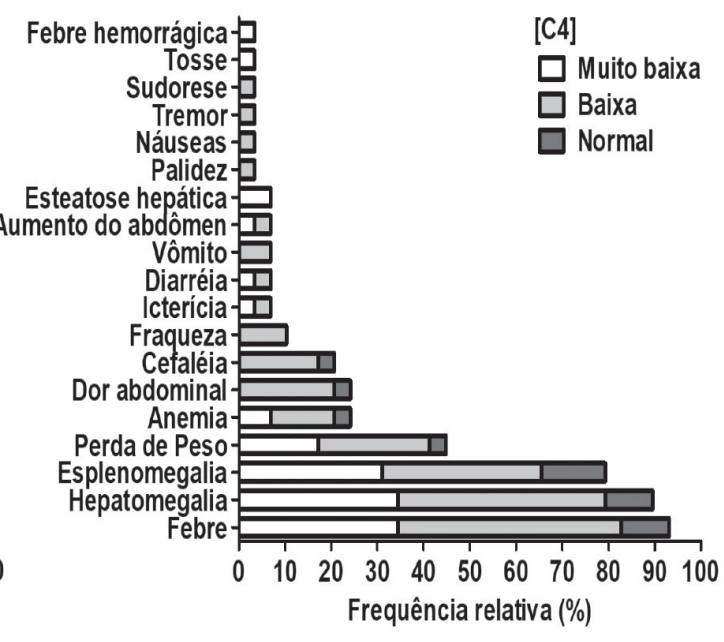

$\mathrm{Na}$ avaliação da série eritrocitária foi demonstrado que a maioria dos pacientes com LV apresentava níveis abaixo do valor de referência para os eritrócitos, hemoglobina, hematócrito, assim como as plaquetas. Quando estratificamos segundo os níveis de C3 e C4, somente o C3 influenciou significativamente nos níveis dos hematócritos, nos quais os pacientes com níveis baixos de $\mathrm{C} 3$ se encontravam mais elevados em relação aos pacientes com níveis normais $(p=0,0406)$. A avaliação de outros parâmetros, como VCM, HCM e CHCM demonstrou que a maioria dos pacientes apresentaram níveis normais, que não foram influenciados quanto aos diferentes níveis de C3 e de C4. A avaliação do RDW demonstrou que uma parte dos pacientes $(n=12)$ apresentaram valores acima valor de referência, independente dos níveis de C3 e de C4 (Figura 3). 
Figura 3 - Avaliação da série eritrocitária e de plaquetas de pacientes com leishmaniose visceral segundo a classificação dos valores inatos de C3 e C4. A comparação entre os diferentes grupos foi realizada pelo teste de Kruskal-Wallis, seguido do teste de Dunn, e foi considerado significante $p<0,05$. Linhas horizontais representam a média e linhas pontilhadas os valores de referência de cada variável.
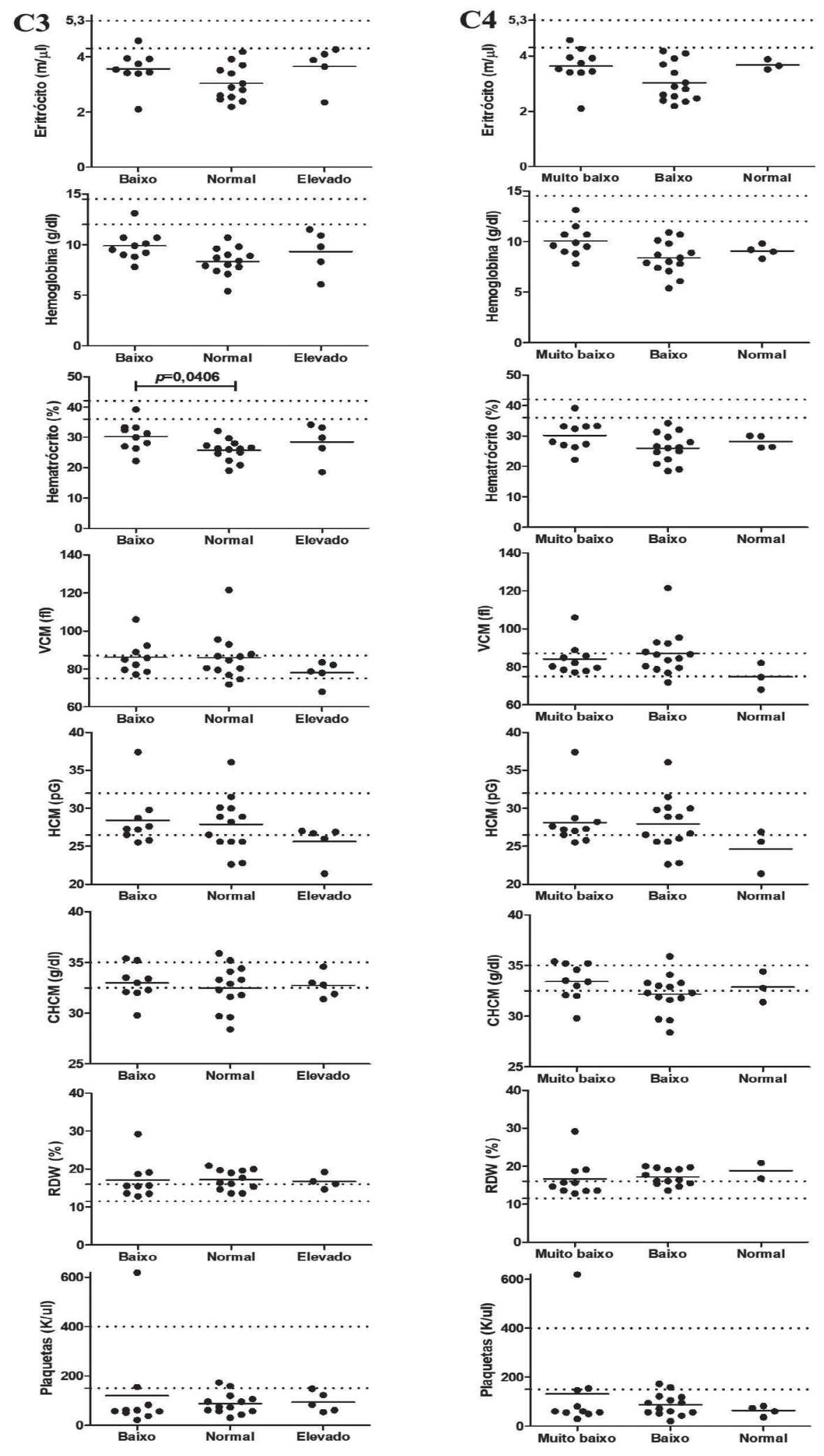

Fonte: Dados da pesquisa. 
A avaliação da série leucocitária demonstrou que a maioria dos pacientes apresentava níveis abaixo do valor de referência para leucócitos, neutrófilos e eosinófilos. A avaliação dos basófilos, linfócitos e monócitos demons- trou que cerca de metade dos pacientes apresentaram níveis normais, que não foram influenciados quanto aos diferentes níveis de C3 e de C4 (Figura 4).

Figura 4 - Avaliação da série leucocitária de pacientes com leishmaniose visceral segundo a classificação dos valores inatos de C3 e C4. A comparação entre os diferentes grupos foi realizada pelo teste de Kruskal-Wallis, seguido do teste de Dunn, e foi considerado significante $p<0,05$. Linhas horizontais representam a média e linhas pontilhadas os valores de referência de cada variável.
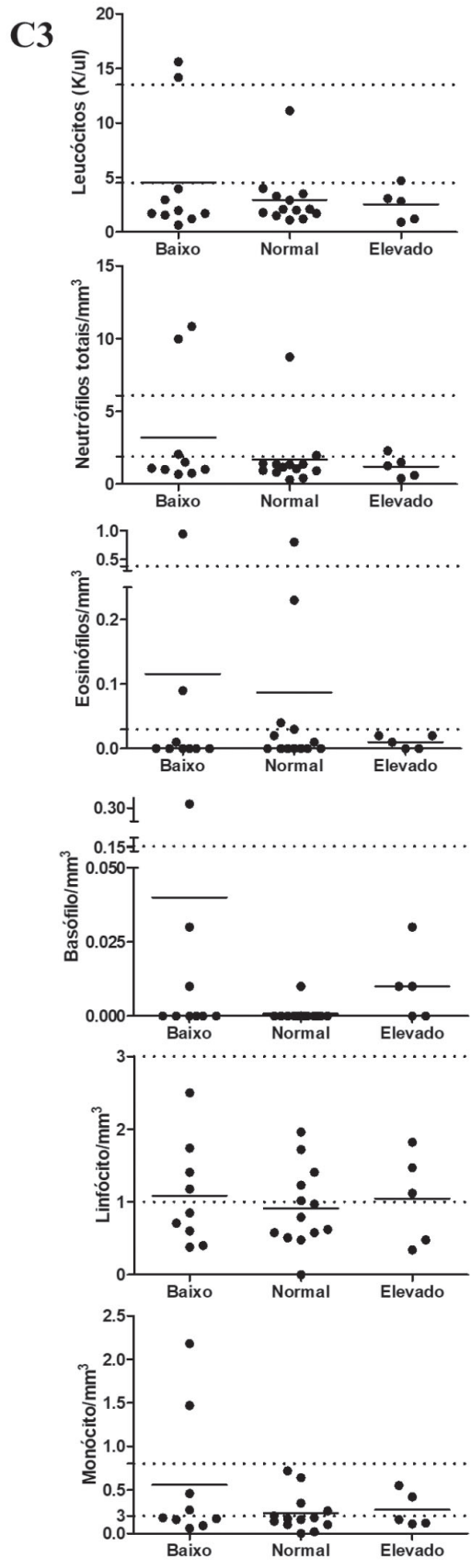

\section{C4}
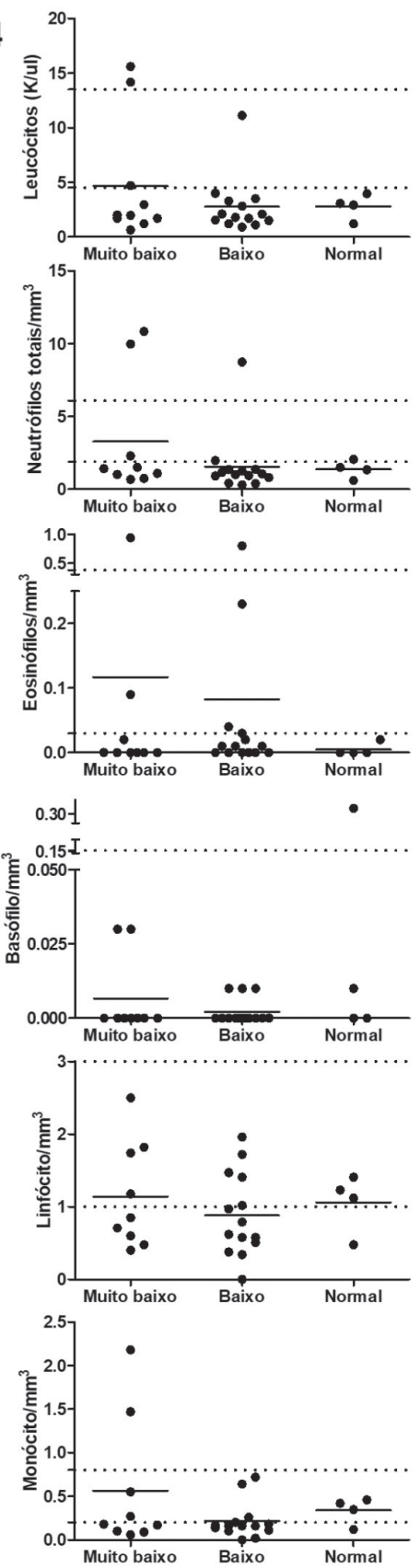

Fonte: Dados da pesquisa. 
níveis de bilirrubina (total, direta e indireta) e creatinina se apresentavam diminuídos e os níveis de potássio, sódio e ureia se apresentavam normais. Poucos pacientes apresentaram valores de TGP normais e a proporção de indivíduos com níveis baixos e elevados foi similar. Os valores dos parâmetros bioquímicos não foram influenciados pelos diferentes níveis de C3 e C4 (Figura 5).

Figura 5 - Avaliação bioquímica de pacientes com leishmaniose visceral segundo a classificação dos valores inatos de C3 e C4. A comparação entre os diferentes grupos foi realizada pelo teste de Kruskal-Wallis, seguido do teste de Dunn, e foi considerado significante $p<0,05$. Linhas horizontais representam a média e linhas pontilhadas os valores de referência de cada variável.
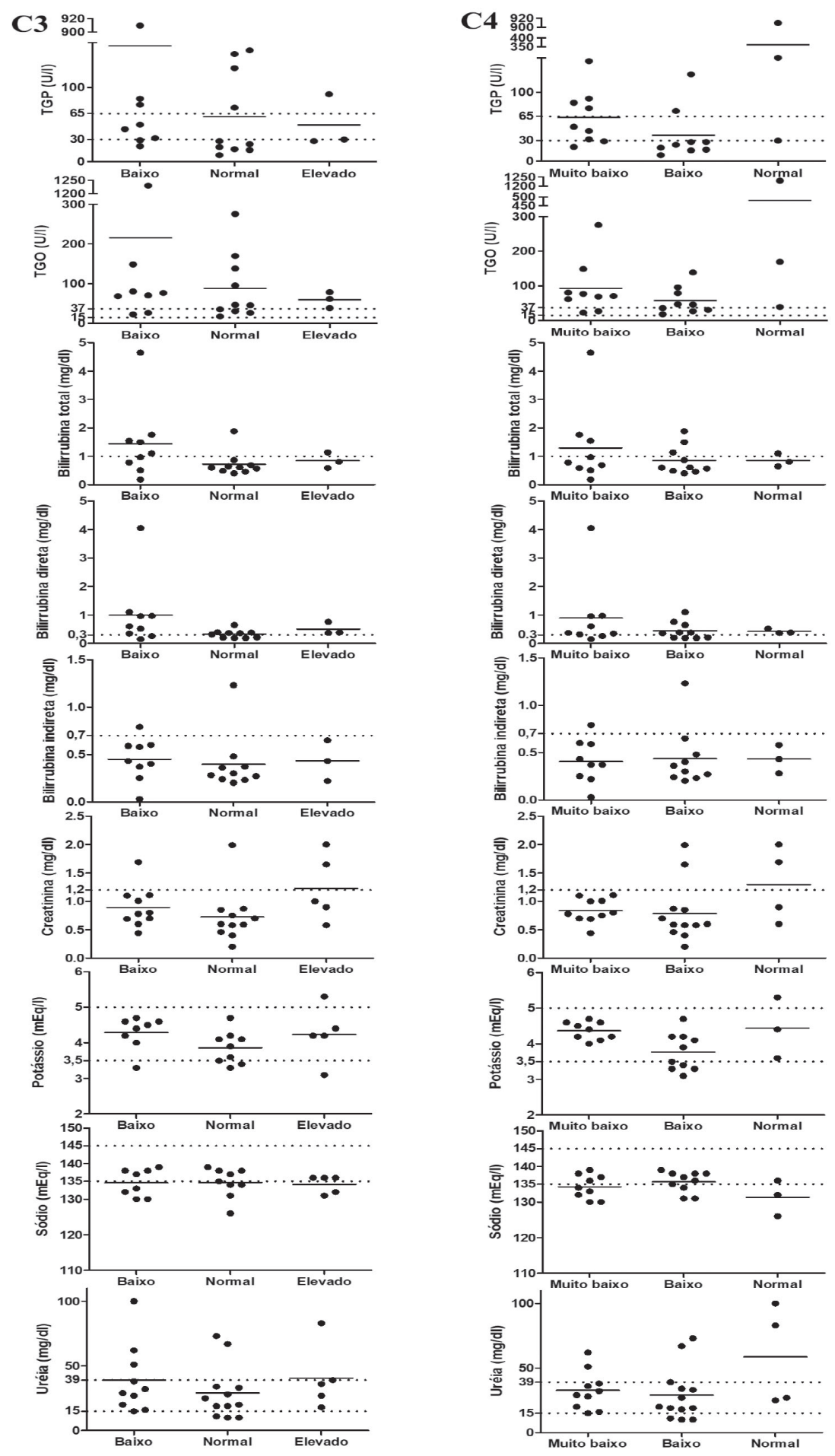

Fonte: Dados da pesquisa. 
A avaliação de parâmetros de fase aguda demonstrou que as proteínas totais se encontravam normais, a albumina baixa, as globulinas elevadas e a relação albumina/ globulina baixa. Os valores não foram influenciados pelos diferentes níveis de C3 e C4 (Figura 6).

Figura 6-Avaliação de marcadores de fase aguda de pacientes com leishmaniose visceral segundo a classificação dos valores inatos de C3 e C4. A comparação entre os diferentes grupos foi realizada pelo teste de Kruskal-Wallis, seguido do teste de Dunn, e foi considerado significante $p<0,05$. Linhas horizontais representam a média e linhas pontilhadas os valores de referência de cada variável.
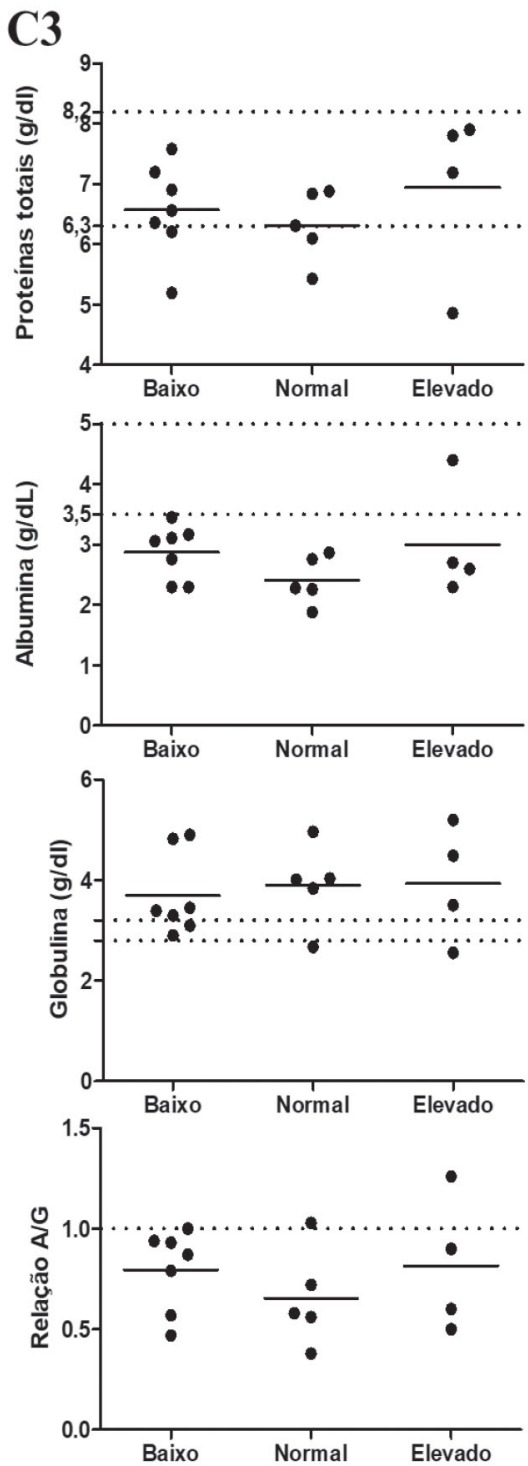

$\mathrm{C} 4$
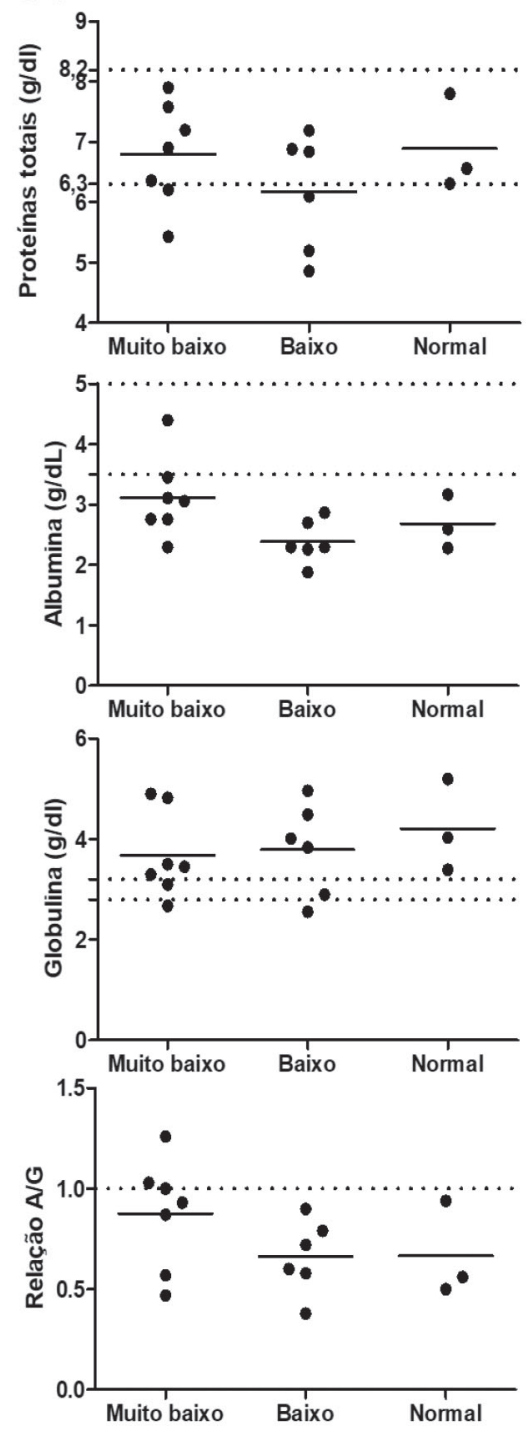

Fonte: Dados da pesquisa.

\section{DISCUSSÃO}

Os pacientes do presente estudo apresentaram idade entre de 19 a 82 anos, e a razão observada entre os sexos foi de 1,6 homens para uma mulher, observando maior frequência em pacientes do sexo masculino, concordando com os dados do Ministério da Saúde, demonstrando que no Brasil os homens são $64,8 \%$ mais afetados que as mulheres. A definição sintomatológica de LV associa-se com hepatoesplenomegalia, anemia, leucopenia, inversão da relação albumina/globulina, trombocitopenia, febre e perda de peso, dados concordantes com o presente estudo ${ }^{11,12}$.

Nossos resultados demonstram que pacientes com LV curada apresentam diferentes níveis inatos de C3 e C4 e não foram encontrados outros estudos que realizaram esta abordagem. A avaliação do C3 apontou a presença de pacientes com níveis elevados deste componente, fato que poderia favorecer a opsonização de promastigotas metacíclicas pelo $\mathrm{C} 3 \mathrm{~b}$, promovendo a fagocitose e induzindo a lise do parasita, pela formação do complexo 
de ataque à membrana (CAM). Entretanto, como mecanismo de escape, a Leishmania apresenta antígenos que são capazes de inativar o C3b (C3bi), impedindo a formação do CAM, o não desencadeamento do estresse oxidativo e diminuição da IL-12, protegendo assim as formas promastigotas ${ }^{13,14}$. Nossos resultados, também demonstraram um grupo com níveis diminuídos de $\mathrm{C} 3$ e de $\mathrm{C} 4$, fato que pode predispor ao desenvolvimento de doenças autoimunes, infecções ou ambas ${ }^{10,15}$.

Desta forma, seria condizente refletir se indivíduos com elevados níveis inatos de C3 teriam uma maior quantidade de C3b formado logo após a inoculação pelo flebotomíneo, fato que poderia facilitar a entrada e persistência da Leishmania no seu hospedeiro. Estudo com camundongos deficientes para CD11b, molécula que atua como receptor de $\mathrm{C} 3 \mathrm{~b}$ e induz a fagocitose, verificou que estes animais eram um pouco mais resistentes à infecção in vivo por L. major, evidenciando o papel do CD11b no desenvolvimento da doença ${ }^{16}$.

Durante a fase aguda da leishmaniose ocorre a desregulação da hematopoese, devido à hiperplasia e elevada carga parasitária presentes na medula óssea é um achado comum em pacientes com LV é a pancitopenia, caracterizada pela presença simultaneamente de trombocitopenia, anemia e leucopenia ${ }^{17}$. Nossos resultados estão de acordo com estes achados, demonstrando diminuição da série leucocitária, entretanto somente seis pacientes apresentaram pancitopenia.

Nossos achados demonstram que a maioria dos pacientes apresentaram anemia com prevalência do tipo normocítica e homocrômica, presença de anisocitose e plaquetopenia, resultados concordantes com estudo realizado no Estado do Ceará ${ }^{18}$. A anemia pode ser associada à inúmeros fatores, dentre os quais, a destruição de eritrócitos, fato que pode estar associado ao componente C3 do sistema complemento, como demonstrado em pesquisa recente, que verificou hemólise via complemento após a sensibilização dos eritrócitos com extrato de leishmania ${ }^{19}$. Nossos resultados demonstraram que indivíduos com C3 baixo apresentavam um hematócrito significativamente mais elevado quando em comparação com indivíduos com níveis normais, demonstrando a associação deste componente com a lesão de hemácias.

Os resultados apresentados demonstraram que pacientes com LV apresentam níveis de TGO e TGP elevados quando associados com níveis normais de C4. Outros estudos demonstraram níveis elevados destas enzimas, entretanto, os mesmos autores verificaram níveis elevados de bilirrubina (direta e indireta) e normais para a creatinina, fato que contradiz nossos resultados, que demonstraram níveis diminuídos de bilirrubina (direta e indireta) e de creatinina ${ }^{18,20}$. Os níveis de ureia no presente estudo demonstraram-se normais, resultados concordantes com outro estudo realizado no Brasil ${ }^{18}$.

A resposta de proteínas de fase aguda é caracterizada por uma reação inflamatória não específica do hospedeiro, e esta resposta tem a função de barrar a ação do patógeno e o dano celular. No presente estudo, os níveis de proteínas totais se encontram normais e não foram influenciados pelos níveis inatos de C3 e C4. A normalidade das proteínas totais foi observada em estudo que avaliou os pacientes com LV logo após o diagnóstico ${ }^{18}$, assim como relatado por Gatto e colaboradores (2013) ${ }^{21}$, que avaliaram pacientes com LV antes e depois do tratamento, não observando diferença entre os diferentes momentos do tratamento.

Não houve influência dos níveis de C3 e C4 sobre as globulinas, que se apresentaram aumentadas em relação ao valor de referência em todos os grupos, fato observado também por outros estudos ${ }^{18,22}$. Estudo que avaliou a produção de gamaglobulinas verificou um aumento em todos os pacientes com LV, associando este achado laboratorial com a ativação policlonal de células B, fato frequentemente encontrado nesta doença ${ }^{22}$.

Alguns marcadores de fase aguda, são conhecidos como negativos, como é o caso da albumina, que se encontram diminuídos durante o processo de fase aguda. Os níveis séricos de albumina estão diretamente relacionados com o estado nutricional do indivíduo e com o sistema imunológico, mostrando que a má nutrição é uma das causas da hipoalbuminemia, além de estar relacionada com o processo infeccioso ${ }^{23}$. Nossos pacientes apresentaram valores diminuídos de albumina, independentemente do valor de $\mathrm{C} 3$ e C4. A hipoalbuminemia também foi relatada em outros estudos ${ }^{12,22}$. Os resultados do presente estudo também demonstraram que a maior parte dos pacientes apresentavam uma acentuada inversão da relação albumina/globulina, característica comum da LV ${ }^{12}$.

\section{CONCLUSÃO}

Concluímos que o componente $\mathrm{C} 3$ do sistema complemento está associado à alterações do hematócrito, sugerindo o acompanhamento dos seus níveis em pacientes com LV. Além disso, ainda não está claro se níveis inatos dos componentes C3 e C4 auxiliam na eliminação ou colaboram com a permanência da Leishmania logo após a entrada no seu hospedeiro, reforçando a necessidade de novos estudos com esta abordagem.

\section{AGRADECIMENTOS}

À Associação Prudentina de Educação e Cultura Apec pelo apoio financeiro.

\section{REFERÊNCIAS}

1. GONTIJO, C.M.F; MELO, M.N. Leishmaniose visceral no Brasil: quadro atual, desafios e perspectivas. Rev. bras. epidemiol., São Paulo, v. 7, n. 3, p. 338-349, Sept. 2004

2. ORGANIZAÇÃO PAN-AMERICANA DA SAÚDE. Leishmanioses: Informe Epidemiológico das Américas. Inf Leishmanioses, [s.I], n. 6. fev. 2018.

3. RUS, H.; CUDRICI, C.; NICULESCU, F. The role of the complement system in innate immunity. Immunol Res., Korea, v.33, n.2, p.103-12, 2005.

4. DUNKELBERGER, J. R.; SONG, W-C. Complement and its role in innate 
and adaptive immune responses. Cell Res., Beijing, v.20, n.1, p. 34-50, 2010.

5. GURUNG, P.; KANNEGANTI, T. D. Innate immunity against Leishmania infections. Cell Microbiol., Oxford, n.17,v.9, p.1286-1294, 2015.

6. GOTO, H.; SANCHEZ, M. C. A. Does the Complement System Work for or Against the Host during Parasite Infections. ITI, [s.I], v.1, n.2, p. $11-23,2013$.

7. HORIMOTO, A.M.C.; DA COSTA, I.P. Frequência de autoanticorpos e dosagem de complemento sérico em pacientes com diagnóstico de leishmaniose cutânea ou visceral. Rev. Bras. Reumatol., São Paulo, v.49, n.5, p.529-546, 2009.

8. LIMONCU, M. E. et al. Complement C1, C3 and C4 levels in sera of leishmaniasis cases in Turkey. Afr. J. Microbiol. Res., Jena, v.5, n.6, p.720-724, 2011.

9. Von STEBUT, E. Immunology of cutaneous leishmaniasis: The role of mast cells, phagocytes and dendritic cells for protective immunity. Eur. J. Dermatol., Mountrouge, v.17, n.2, p.115-22, 2007.

10. AUDEMARD-VERGER, A. et al. Infections revealing complement deficiency in adults: A French nationwide study enrolling 41 patients. Med., United States, v.95, n.19, p.1-4, 2016.

11. BRASIL. Ministério da Saude. Situação epidemiológica da Leishmaniose visceral em 2017. Brasília: Secretaria de Vigilância em Saúde, 2017.

12. BRASIL. Ministério da Saúde. Doenças infecciosas e parasitárias: Guia De Bolso. Brasília: Departamento de Vigilância Epidemiológica, Secretaria de Vigilância em Saúde, 2010.

13. COULTHARD, L.G.; WOODRUFF, T.M. Is the Complement Activation Product C3a a Proinflammatory Molecule? Re-evaluating the Evidence and the Myth. J. Immunol., Rockville, v.194, n8, p.3542-8, 2015.

14. SANTOS-MATEUS, D. et al. The Battle between Leishmania and the Host Immune System at a Glance. ITI, [s.I], v.4, n.1, p.3121-2326, 2016.
15. RAM, S., LEWIS, L.A.; RICE; P.A. Infections of people with complement deficiencies and patients who have undergone splenectomy. Clin Microbiol Rev., Cambridge, v.23, n.4, p.740-780, 2010.

16. CARTER, C. R. et al. Complement receptor 3 deficiency influences lesion progression during Leishmania major infection in BALB/c mice. Infect. Immun., Washington, v. 77,p. 5668-5675, 2009.

17. MOREIRA, E.A. Aspectos Hematológicos De Pacientes Com Leishmaniose Visceral. Academia de Ciência e Tecnologia, São José do Rio Preto, Março, p.1-9, 2012.

18. PRAZERES, J.L. et al. Investigação laboratorial de pacientes adultos com leishmaniose visceral de um hospital público de Fortaleza, no período de 2007-2008. Rev. Ciênc. Méd. Biol., Salvador, v.12, n.2, p.173-179, 2013.

19. Lemos, A.F.B. Avaliação da ativação do sistema complemento por extratos proteicos de Leishmania spp.. 2016. Dissertação (Mestrado em Ciências Biomédicas) - Universidade Nova de Lisboa, Instituto de Higiene e Medicina Tropical, Lisboa, 2016.

20. MATHUR, P.; SAMANRATY, J.C.; SAMANTA, P. High prevalence of functional liver derangement in Visceral Leishmaniasis at an Indian Tertiary Care Center. Clin. Gastroenterol. Hepatol., Philadelphia, v.6, n.10, p.1170-1172, 2008.

21. GATTO, M. et al. Biochemical and nutritional evaluation of patients with visceral leishmaniasis before and after treatment with leishmanicidal drugs. Rev. Soc. Bras. Med. Trop., Uberaba, v.46, n.6, p.735-740, Dec. 2013a.

22. SALGADO FILHO, N.; FERREIRA, T.M.A.F.; COSTA, J.M.L. Envolvimento da função renal em pacientes com leishmaniose visceral (calazar). Rev. Soc. Bras. Med. Trop., Uberaba, v.36, n.2, p.217-221, 2003.

23. BROCK, F. et al. Prevalence of hypoalbuminemia and nutritional issues in hospitalized elders. Rev. Latino-Am. Enfermagem, Ribeirão Preto, v.24, e2736, 2016.

Submetido em: $15 / 12 / 2019$

Aceito em: 27/05/2020 